\title{
Weighted k-Nearest Neighbor Adaptations to Spare Part Prediction Business Scenario at SAP System
}

\author{
Eren Esgin \\ AI Research, MBIS R\&D Center, Istanbul, Turkey \\ Informatics Institute, Middle East Technical University, Ankara, Turkey
}

\begin{abstract}
Keywords: Classification, CRISP-DM, Intelligent Maintenance, SAP, Spare Part Prediction, Weighted k-Nearest Neighbor.

Abstract: In the context of intelligent maintenance, spare part prediction business scenario seeks promising return-oninvestment (ROI) by radically diminishing the hidden costs at after-sales customer services. However, the classification of class-imbalanced data with mixed type features at this business scenario is not straightforward. This paper proposes a hybrid classification model that combines C4.5, Apriori algorithms and weighted kNearest Neighbor $(\mathrm{kNN})$ adaptations to overcome potential shortcomings observed at the corresponding business scenario. While proposed approach is implemented within CRISP-DM reference model, the experimental results demonstrate that proposed approach doubles the human-level performance at spare part prediction. This highlights a $50 \%$ decrease at the average number of customer visits per fault incident and a significant cutting at the relevant sales and distribution costs. According to best runtime configuration analysis, a real-time spare part prediction model has been deployed at the client's SAP system.
\end{abstract}

\section{INTRODUCTION}

Average number of customer visits per fault incident is a critical key performance indicator (KPI) at aftersales customer services such that, undesirable repetitive customer visits result in a significant increase at hidden sales and distribution costs. Additionally, it may affect the quality level of aftersales services and deteriorates the organizational goodwill at long run. Respectively, spare part prediction business scenario aims to generalize the spare part consumption patterns according to failure characteristics, product's own features and consumer detailed information and then proactively proposes the most probable spare part for new failure incident.

Although classification algorithms have been widely used in retail, finance, banking, security, astronomy and behavioral ecology domains (Kantardzic, 2011) and the classifiers for classbalanced data are relatively well developed, the classification of class-imbalanced data with mixed type features is not straightforward (Liu et al., 2014). This paper proposes a hybrid classification algorithm such that, while Apriori is adapted to handle data anomalies and redundancies observed at data preparation, significance weights obtained at $\mathrm{C} 4.5$ are used for doing normalization on categorical features to adapt the inter-dimension similarity at computing the similarity between fault instances. As the following, two adaptations of weighted $\mathrm{kNN}$ are applied: while instance based $k N N$ with count (IkNNwC) gives more importance to major instances that are more likely to represent a dominant class in neighborhood region of feature space, instance based $k N N$ with average similarity score (IkNNwAS) aims to balance the discriminative power of minor (or outlier) instances. Proposed approach is evaluated according to the fault records of television (TV) product group within 5 years' time period and fullcycle data mining framework, which covers all phases from business understanding to deployment, is implemented according to CRISP-DM (Cross Industry Standard Procedure for Data Mining) reference model.

The paper is organized as follows. Section 2 reviews the related work about $\mathrm{kNN}$ adaptations. Section 3 explains the proposed classification approach within the context of CRISP-DM reference model such that, business understanding, data understanding, data preprocessing and modeling phases are briefly explained. Section 4 discusses the experimental results according to the performance 
observed at evaluation and deployment phases. The conclusion and future work are summarized in Section 5 .

\section{LITERATURE REVIEW}

$\mathrm{kNN}$ algorithm considers a firm representative of the classification by analogy (Domingos, 2015). Naturally, finding an optimal value of $k$, which represents how many closest neighbors are to be considered, has been one of the questions that some works have attempted to solve (Zhang et al., 2017; Zhu et al., 2016). Besides finding the $\mathrm{k}$-value, the underlying distance calculation is another issue in this kind of classification. Using a weighted scheme was first introduced by (Dudani, 1976), this variant of kNN is called Distance-Weighted k-Nearest Neighbor (DWkNN). (Tan, 2015) proposed the algorithm Neighbor-Weighted k-Nearest Neighbor (NWkNN), which applies a weighing strategy based on the distribution of classes. (Mateos-Garcia et al., 2016) developed a technique that optimizes the weights that would indicate the importance of neighborhood in a similar way of Artifical Neural Network. (Parvinnia et al., 2014) also computed a weight for each training object based on a matching strategy. Respectively, (Aguilera et al., 2019) proposed a weighting based on Newton's gravitational force, so that a mass (or relevance) is to be assigned to each instance. Two methods of mass assignment is presented: circled by its own class (CC) and circled by different class (CD).

The standard kNN algorithm is not suitable for the presence of imbalanced class distribution. Hence, kENN in (Yuxuan \& Zhang, 2011) and CCW-kNN in (Liu \& Chawla, 2011) have been proposed to improve the performance of $\mathrm{kNN}$ for imbalance classification. While kENN proposed a training stage where positive training instances are identified and generalized into Gaussain balls, CCW-kNN uses the probability of feature values given class labels to weight prototypes in kNN. (Song et al., 2007) also proposed new kNN algorithms based on informativeness which is introduced as a query-based distance metric. This informativeness is handled in two concerns: locally informative (LI-kNN) and globally informative (GIkNN). Alternatively, (Wang et al., 2011) presented a coupled nominal similarity to examine both intra- and inter-coupling of categorical features. These approaches majorly focused on the clustering on class-balanced data.

\section{PROPOSED APPROACH}

CRISP-DM reference model is applied as the major road map for spare part prediction scenario. Respectively, the underlying sequence of the phases is not rigid, moving back and forward between difference phases is always required (Chapman et al., 1999). CRISP-DM reference model consists of six phases: business understanding, data understanding, data preparation, modelling, evaluation and deployment. Except evaluation and deployment phases, we briefly outline corresponding phases at the following sections.

\subsection{Business Understanding}

In current (as-is) situation, each customer call to customer call center triggers a new fault record at SAP CRM system. During this call, fault occurrence details (e.g. product group, complaint or symptom information in a hierarchical manner) are gathered from the customer. Then, customer details (e.g. customer profile and location) are enhanced and product details (e.g. product SKU (stock keeping unit), material type, material group and product hierarchy) are extracted from prior product assembly history at SAP CRM system. Afterwards, the corresponding fault record is assigned to a near-by technical service according to customer's location. Finally, the technical service makes a feasibility visit to check out the fault reason and defective component. Each customer visit for the corresponding fault incident is managed by a unique maintenance line item and spare part consumption or maintenance activity at this customer visit is charged to this line item.

As the to-be situation, it is aimed to position a spare part prediction model that suggests the most probable spare part for the corresponding fault incident and passes this suggestion to the technical service in a real-time manner. Hence technical service can proactively reorganize the in-car spare part stock and daily customer routes. Moreover, it is aimed to radically diminish average number of customer visits per fault incident. Indeed, hidden sales and distribution cost items and spare part consumptions are strongly correlated to the number of customer visits and reductions at the corresponding KPI will minimize relevant expenses at income statement (e.g. freight costs, maintenance and depreciation costs of technical service vehicles, etc.). As an intangible outcome, we also aim to improve the quality level of after-sales services and increase the organizational 
goodwill in long run. Current as-is and proposed tobe situations are represented in Figure 1.

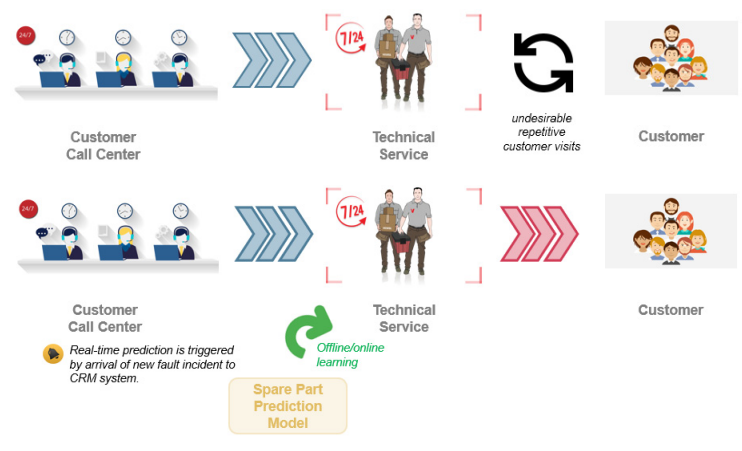

Figure 1: Current (as-is) and to-be scenarios. By the effect of spare part prediction model, undesirable repetitive customer visits will be lessened.

As the human-level performance in the current as-is situation, the average number of customer visits per fault incident KPI is approximately 2.5 , which means a $40 \%$ accuracy at predicting the appropriate spare part. The data mining objective in this business scenario is $80 \%$ accuracy and this implies halving of relevant sales and distribution costs.

\subsection{Data Understanding}

Data understanding phase starts with describing major data sources, the relations among these data sources and major attributes that build up the initial raw data. At first, the data dictionary enlisting all gross and surface properties of the initial raw data is described. Then, the corresponding data description is explored to assess potential anomalies and data redundancies among the attributes and verify data quality problems in order to refine the initial raw data.

\subsubsection{Data Description}

The corresponding business scenario is composed of five data sources:

- Fault Incident. Fault incident holds the header information of corresponding incident record, e.g. fault incident ID, incident date and time, symptom codes, document status, relevant customer ID and product SKU.

- Maintenance Line Item. Maintenance line item holds spare part consumption and maintenance activity charged at each customer visit. There exists a one-to-many $(1: \mathrm{N})$ relation between fault incident and maintenance line item.

- Product. Product holds the major features about the defective product, e.g. product SKU, material type, material group, product hierarchy, brand and product costing group.

- Product Details. Product details holds major production details, e.g. production date and warranty beginning date. There exists a one-toone $(1: 1)$ relation between product and product details data sources.

- Customer. Customer holds the customer profile and location in a city-to-district hierarchy.

Context diagram given in Figure 2 depicts the relations among the data sources.

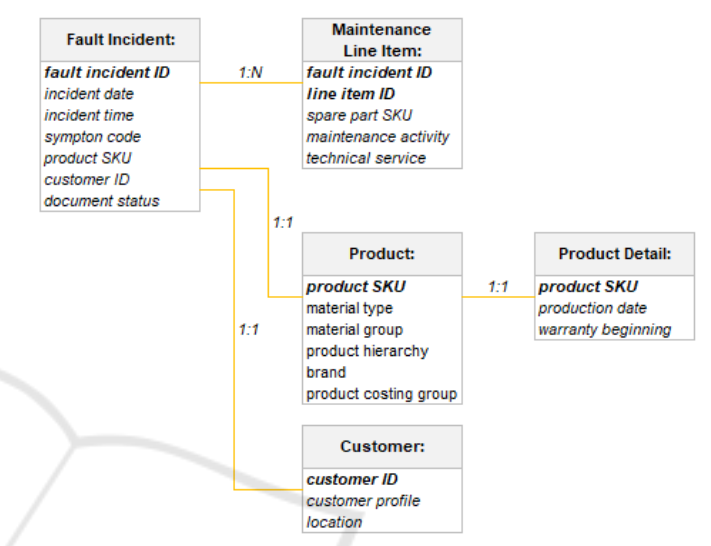

Figure 2: Context diagram for the corresponding data sources.

\subsubsection{Data Exploration}

Data exploration assesses the correlation among the attributes and checks whether any data anomalies and redundancies occur. According to these assessment actions, while each instance at initial raw dataset represents a unique maintenance line item, a significant data replication problem has emerged such that; except the spare part target class, all attributes are acquired from the same data sources, i.e. fault incident, product, product detail and customer. As a result, there occurs distinct instances featured with replicated (the same) attribute values and distinct spare part target value at initial raw data collection. As a solution, each instance should be characterized at a higher abstraction level by relating to a unique fault incident. Hence, an alternative data exploration procedure is applied to collect the spare part target class as shown in Figure 3.

Accordingly, alternative data exploration procedure is composed of three steps as follows:

- Raw Data Aggregation. This initial step aggregates the spare part consumptions at each maintenance line item that are relevant to the same fault incident. Respectively, it resembles transposing the spare part values at relevant 


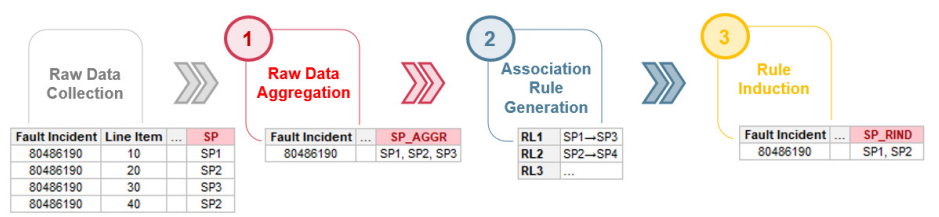

Figure 3: Alternative data exploration procedure. A fault incident with four maintenance lines is transformed into a single instance at final dataset.

maintenance line items and concatenating these values into a single derived target class, i.e. SP_AGGR. While concatenating spare part values at a fault incident, duplicated values are unified and unique values are sorted in ascending order, e.g. $<\mathrm{SP} 1, \mathrm{SP} 1, \mathrm{SP} 3, \mathrm{SP} 2>$ is aggregated as $<\mathrm{SP} 1$, $\mathrm{SP} 2, \mathrm{SP} 3>$.

- Association Rule Generation. Respectively, aggregated dataset is like frequent itemsets and these itemsets can be represented by a Boolean vector of spare part values to underlying variables. Indeed, these Boolean vectors can be analyzed for spare part consumption patterns that highlight frequently associated spare part combinations. These patterns can be represented in the form of association rules.

Apriori is a seminal algorithm proposed for frequent itemsets for Boolean association rules. The name of the algorithm is based on the fact that it uses prior knowledge of frequent itemset properties (Kantardzic, 2011). In this context, Apriori is applied by using $\mathrm{R}$ ( $\mathrm{R}$ packages: arules and arulesviz). Then generated association rules are filtered by min_lift, i.e. min_lift $>1.0$ threshold is used to extract only positively correlated spare part combinations.

- Rule Induction. Rule induction step converts aggregated spare part target class values (SP_AGGR) into refined forms according to previously generated association rules.

In this aspect, significant association rules are determined by min confidence threshold, the default value of this parameter is 0.8 . Filtered association rules are sorted by lift and confidence values in descending order. Afterwards, each spare part target class value at aggregated data set is searched at association rules whether antecedent and consequent of the association rule both exist at the corresponding aggregated spare part target class value. In the case of presence, consequent is removed from the aggregated spare part target class value and this new value is assigned to a new target class, i.e. rule induced spare part SP_RIND. Otherwise, original value of aggregated spare part is copied to rule induced spare part target class.
Figure 4 shows the effect of rule induction step at the frequencies of target classes, i.e. SP_AGGR and SP RIND. Due to $<320001053>\rightarrow<303113320>$ association rule, there happens a significant increase at the frequency of 320001053.

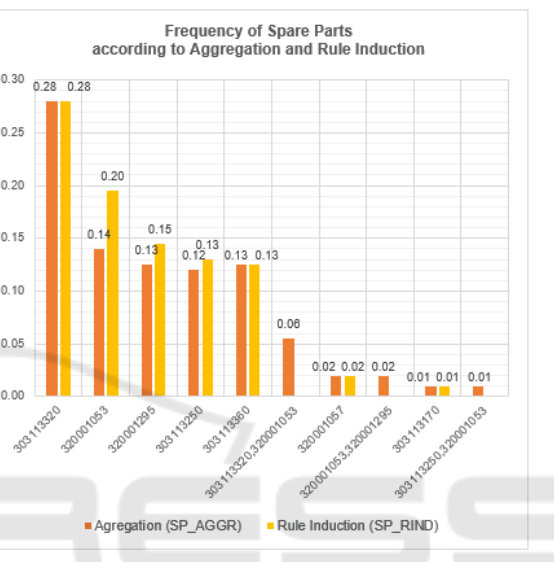

Figure 4: Frequency of spare parts according to aggregation and rule induction operations.

\subsection{Data Preparation}

Data preparation covers data integration, transformation and cleaning activities that are required to construct the final dataset from the initial raw data.

In data integration step, an appropriate SQL script according to the context diagram given in Figure 2 is implemented to extract the fault incidents of television (TV) product group, which occurred within 5 years' time span (between year 2014 and 2018) from SAP CRM and SAP BW source systems,. Then alternative data exploration procedure stated in Section 3.2.2 is applied to avoid data replications and anomalies observed at maintenance line item level. Due to raw data aggregation step at the underlying procedure, a vertical data reduction occurs such that; $750 \mathrm{~K}$ instances at maintenance line item dataset are suppressed to $350 \mathrm{~K}$ instances at aggregated final dataset. Additionally, in order to avoid attribute redundancy due to hierarchical (ordinal) attributes (e.g. customer location, product hierarchy and symptom codes), attributes with relatively higher detail level and wider value range are selected. For 
instance, product hierarchy 8 attribute (PRDHYR8) is selected as the most detailed product hierarchy feature and lower level attributes (PRDHYR2-7) are omitted. At data transformation step, month and year attributes are parsed from the underlying date typed attributes, e.g. incident date and production date. Moreover, new attributes such as product age, product stock age and warranty status are derived.

Indeed, the value range of rule induced spare part target class (SP_RIND) is composed of 1267 distinct values. Hence, the instances with relatively less frequent spare part values (i.e. freq(RIND_SP) $<1000$ condition refers to a $0.22 \%$ frequency) are eliminated at data cleaning step and a $95.27 \%$ total coverage at final dataset is achieved after this operation as shown at frequency histogram given in Figure 5. Additionally, various spare part groups are defined according to the frequency order such as ALL, TOP3 and TOP6 such that, TOPX implies topmost $X$ spare parts according to the frequency at the final dataset. The underlying histogram highlights classimbalanced dataset rationale.

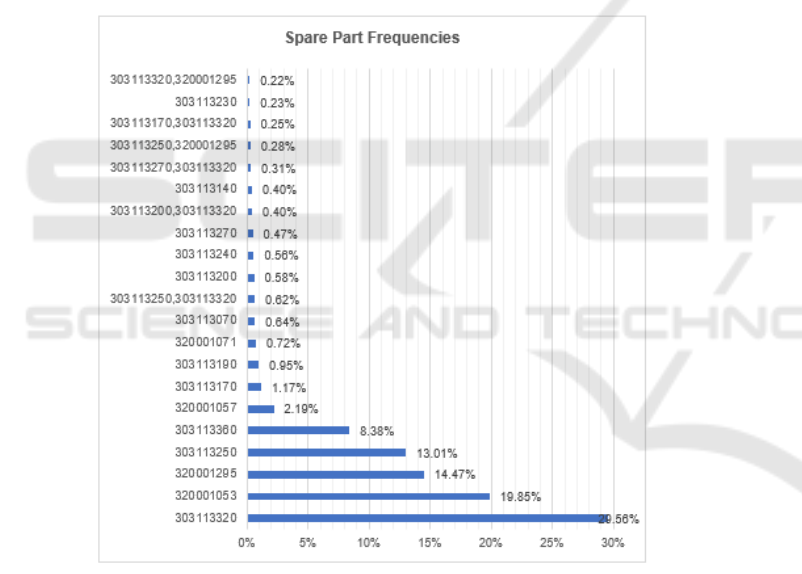

Figure 5: Frequency of spare part target values after data cleaning.

\subsection{Modelling}

Spare part prediction business scenario is a kind of supervised learning due to the existence of a target class, SP_RIND, and the major objective of this scenario is to seek significant drivers and patterns highlighting the underlying phenomenon. According to data dictionary, almost all attributes at final dataset are categorical with a wide value range except the derived attributes, e.g. product age and product stock age. Therefore, we proposed a hybrid approach that combines $\mathrm{C} 4.5$ and Apriori algorithms with weighted $\mathrm{kNN}$ adaptations for the underlying class-imbalanced mixed type final dataset.

\subsubsection{C4.5}

C4.5 adapts a greedy and nonbacktracking approach in which decision trees are constructed as the classifier in a top-down recursive divide-and-conquer fashion (Kantardzic, 2011). The corresponding attribute selection method specifies a heuristic procedure for selecting the attribute that best discriminates the given tuples according to class.

In the context of spare part prediction scenario, C4.5 is applied by using R (R package: rpart) with information gain attribute selection and min_split parameter is set as 50 . While $42.5 \%$ accuracy performance of $\mathrm{C} 4.5$ suggests a ground truth for the candidate algorithms, it majorly proposes the significance weight of the attributes at determination of spare parts as shown in Figure 6. Although several similarity measures, such as the Jaccard coefficient overlap (Pang-Ning et al., 2006), cosine similarity (Liu et al., 2014) and Goodall similarity (Boriah, 2008) can be used with categorical data, they are usually general as similarities at continuous data and ignores the information hiding in the co-occurrence with the target class. Hence, significance weight obtained by C4.5 are used as inter-coupling similarity weights (interDim_weight) at kNN adaptations as given in Section 3.4.3.

Figure 6: Radar graph for significance weight of attributes according to C4.5. Respectively, product hierarchy 8 and symptom code are the main determinants at spare part prediction.

\subsubsection{Apriori}

As stated in Section 3.2, Apriori fundamentally explores significant association and correlation rules among spare part consumptions. The underlying algorithm also generates IF/THEN typed causality rules for predicting target class without presence of a classifier. Respectively, Apriori is applied with min_support $>0.01$ condition for the final dataset and approximately 2710 causality rules are generated. Table 1 exemplifies some generated Apriori causality rules. 
Table 1: Sample IF/THEN typed causality rules generated by Apriori.

\begin{tabular}{|c|c|c|c|c|}
\hline Predicate & Target & Support & Confidence & Lift \\
\hline ZPRDAGE_GR=G1,ZSIIKAYET_T=S949285 & 303113250 & 0.0100 & 0.9248 & 6.2660 \\
\hline ZGRNTDHL $=Y, Z$ ZSIKAYET_T=S949285 & 303113250 & 0.0225 & 0.9147 & 6.1976 \\
\hline MEVSIM=SONBAHAR,ZPRDAGE_GR=G0 & 303113320 & 0.0326 & 0.4034 & 1.2671 \\
\hline ZPRDAGE_GR $=$ G0,ZURTMONTH_YR_T $=Y 2015$ & 303113320 & 0.0284 & 0.4155 & 1.3053 \\
\hline BOLGE $=$ MARMARA ZPRDAGE $-\overline{G R}=\bar{G} 0$ & 303113320 & 0.0306 & 0.4508 & 1.4161 \\
\hline
\end{tabular}

Rules with min_lift $>1.0$ property are validated according to 66 test scenarios, which are configured by different incident year, spare part groups (ALL, TOP3 and TOP6) and validation methods (i.e. hold-out and $k$-fold cross-validation with $k=3,5,10)$. While ALL year group has an average accuracy of $52 \%$, the accuracy for year 2017 peaks at $55.7 \%$. The lowest average accuracy of $45.3 \%$ is obtained at year 2015 . Moreover, a significant correlation between rule precision and confidence is observed as shown in Figures 7. However, rules with confidence values between 0.4 and 0.6 seriously result in false predictions (FP-false positive) with an average precision of $25.27 \%$.

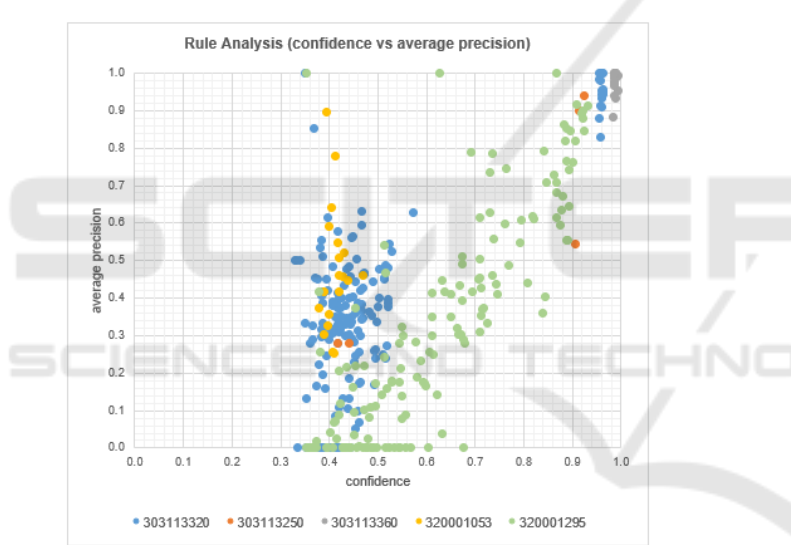

Figure 7: Rule analysis (confidence vs average rule precision).

According to Table 2, min confidence $=0.7$ is designated as the confidence threshold for filtering relatively weak causality rules. Figure 8 emphasizes the average precision gap between the rule groups.

Table 2: Confidence threshold determination.

\begin{tabular}{lccccc}
\hline & \multicolumn{6}{c}{ confidence threshold } \\
\hline & 0.60 & $\mathbf{0 . 7 0}$ & 0.80 & 0.90 & 1.00 \\
\hline avg precision & $67.26 \%$ & $\mathbf{7 6 . 0 5 \%}$ & $84.20 \%$ & $93.27 \%$ & $0.00 \%$ \\
number of rules & 115 & $\mathbf{9 0}$ & 68 & 44 & 0 \\
\hline
\end{tabular}

\subsection{3 kNN and Adaptations}

$\mathrm{kNN}$ classification is based on online learning scheme by analogy; that is by comparing a given test instance with training tuples at knowledge repository that are similar to it (Kantardzic, 2011). The training tuples are

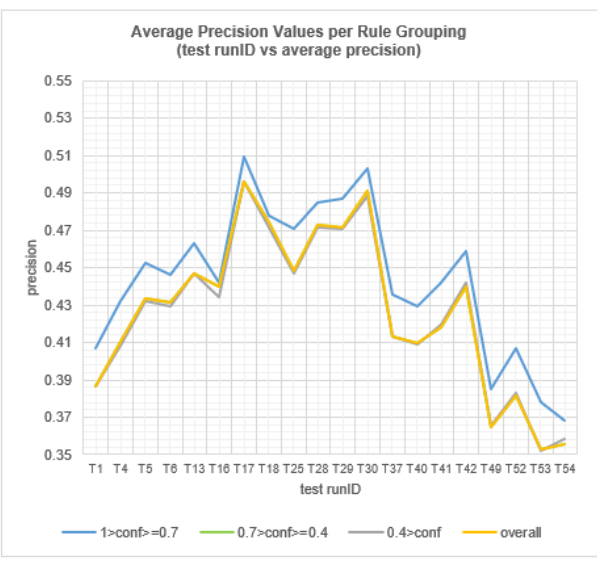

Figure 8: Average precision values per rule grouping.

represented in a $n$-dimensional pattern space. When given an unknown tuple, a $\mathrm{kNN}$ classifier searches the pattern space for the $k$ training tuples that are closest to the unknown instance. Closeness is defined in terms of a distance function such as Euclidean distance. Typically, the values of each attribute should be normalized before distance calculation. But the distance calculation for categorical attributes and relative distance within the value range of these attributes are two major issues emerged at spare part prediction scenario. As the first adaptation to $\mathrm{kNN}$, the underlying closeness measurement is converted into a similarity measurement, $\operatorname{simScr}\left(\right.$ ins $_{i}$, ins $\left._{j}\right)$, as shown in Equation 1.

$$
\operatorname{simScr}\left(\text { ins }_{i}, \text { ins }_{j}\right)=\sum_{\text {dim }=1}^{n} \begin{gathered}
\text { interDim_weight } \\
\text { intraDim_weight }\left(\text { ins }_{i} \text {.dim, } \text { ins }_{j} \text {.dim }\right)
\end{gathered}
$$

In Equation 1, interDim_weight is the normalization weight assigned for each significant attribute ( $\mathrm{dim})$ and the significance weight obtained at C4.5 is used for normalizing categorical attributes to adapt the inter-dimension similarity. intraDim_weight factor holds the similarity degree of different level of the corresponding hierarchical (ordinal) attributes. While these similarity degrees are determined by domain experts, nominal difference at the numeric attribute values of instances, ins $s_{i}$ and $i n s_{j}$, is used as intraDim_weight. In the context of similarity measurement, we propose two kNN adaptations: instance based $k N N$ (IkNN) and average $k N N(\mathrm{AkNN})$.

At IkNN adaptation, the similarity between new fault incident and neighboring objects in the final dataset is measured by Equation 1. Then the nearest neighboring data points according to the similarity values are determined by neigh_limit. This argument is a percentile limit that preserves the closest training objects in a spherical-like region and its value is 
parametrized at $[0.2 \%, 1.0 \%]$ interval. Then as shown in Figure 9, count, total and average similarity values per spare part target class value are summarized within the closest neighboring objects. At this point, IkNN has two variations:

- Instance based kNN with Count (IkNNwC). Common spare parts at prediction result list are determined by count value. By doing this, it is aimed to give less importance to objects that are more likely to represent a different class. In other words, the idea is to penalize rare instances and make the classifier more robust to the outliers.

- Instance based kNN with Average Similarity Value (IkNNwAS). Common spare parts at prediction result list are determined by average similarity value. This variation aims to balance the discriminative power of an outlier object, since it could be relevant to classify other outlier object. It also allows to better modeling classimbalanced dataset by giving more chance to objects less represented.

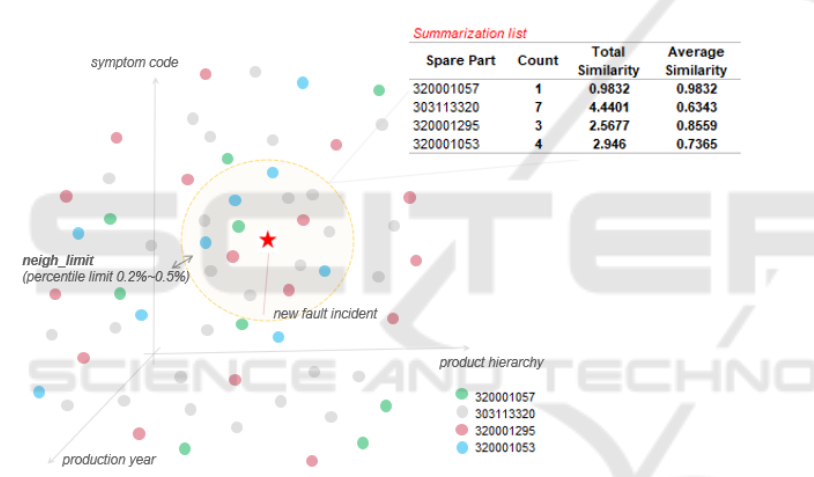

Figure 9: A sample Instance based kNN (IkNN) use-case for a new fault incident.

Finally, spare part prediction result list is finalized according to $k$-limit argument as follows:

- In the case of $k$-limit $=n$, i.e. $n=1,2 \ldots$, the topmost $n$ spare part values according to count or average similarity value rank are returned as prediction result list. The maximal value for $n$ is limited as 2 at this business scenario.

- In the case of dynamic $k$, i.e. $k$-limit $=\mathrm{DK}$ and DK in $[0,1]$ interval, the standard deviation $(s t d D e v)$ of the corresponding value (i.e. count or average similarity) is calculated. If the difference between two consecutive spare part target values is greater than $\mathrm{DK} \times$ stdDev, then prediction result list is returned as the combination of all checked spare part values. Otherwise, it is continued to check the following lines at summarization list.

Table 3 exemplifies the $k$-limit application at IkNN variation.
Table 3: k-limit application for IkNN variations. Especially larger DK values with lower standard deviation may weaken the capability at selective prediction. Hence, longer prediction result list results in an accuracy decrease.

\begin{tabular}{|c|c|c|c|c|c|c|}
\hline \multicolumn{3}{|c|}{ Instance based KNN with count } & \multicolumn{4}{|c|}{ dynamic $k$} \\
\hline Spare Part & Count & $k=1$ & $k=2$ & $D K=1.0$ & $\mathrm{DK}=0.5$ & $\mathrm{DK}=0.25$ \\
\hline 303113320 & 7 & $\sqrt{ }$ & $\sqrt{ }$ & $\sqrt{ }$ & $\sqrt{ }$ & $\sqrt{ }$ \\
\hline 320001053 & 4 & & $\sqrt{ }$ & & & \\
\hline 320001295 & 3 & & & & & \\
\hline 320001057 & 1 & & & & & \\
\hline \multicolumn{7}{|l|}{ stdDev:2.165 } \\
\hline \multicolumn{5}{|c|}{ Instance based KNN with average similarity } & \multicolumn{2}{|c|}{ dynamic $k$} \\
\hline Spare Part & $\begin{array}{l}\text { Average } \\
\text { Similarity }\end{array}$ & $k=1$ & $k=2$ & $\mathrm{DK}=1.0$ & $\mathrm{DK}=0.5$ & $\mathrm{DK}=0.25$ \\
\hline 320001057 & 0.9832 & $\sqrt{ }$ & $\sqrt{ }$ & $\sqrt{ }$ & $\sqrt{ }$ & $\sqrt{ }$ \\
\hline 320001295 & 0.8559 & & $\sqrt{ }$ & $\sqrt{ }$ & & \\
\hline 320001053 & 0.7365 & & & $\sqrt{ }$ & & \\
\hline 303113320 & 0.6343 & & & $\sqrt{ }$ & & \\
\hline \multicolumn{7}{|c|}{ stdDev:0.1305 } \\
\hline
\end{tabular}

Respectively, AkNN is similar to IkNN adaptation except neighbor preservation such that, neigh_limit argument is not applied at AkNN. Otherwise, summarization list is formed by traversing all training tuples at the final dataset. Therefore, AkNN is relatively more time-consuming and rather less capable at pinpointing minor (or outlier) objects at $n$ dimensional space.

\section{EXPERIMENTAL RESULTS}

Major outcomes of evaluation and deployment phases at CRISP-DM life cycle are presented in this section.

\subsection{Evaluation}

As stated in Section 3.4.2, Apriori causality rules with confidence between 0.4 and 0.6 tend to make erroneous predictions such that, they have an average precision of $25.27 \%$. Therefore, min_confidence $=$ 0.7 is designated as confidence threshold to eliminate these weak rules. Respectively, we propose the following hybrid classification algorithm:

> Initially, it is attempted to predict new fault incident by relatively confident Apriori causality rules.

$>$ In the case of unpredicting by Apriori, kNN adaptations are applied to classify the corresponding incident by an online learning schema.

Hence, 56 test scenarios are configured by varying incident year, spare part groups, kNN variations and arguments (neigh_limit and $k$-limit) and validation methods. As shown in Table 4, while pure Apriori 
casuality rules have an average accuracy of $54.02 \%$, the combination of Apriori with IkNN adaptation improves average accuracy towards $77.95 \%$ level. This metric is approximately $85.2 \%$ for TOP 3 and $79.24 \%$ for TOP6 spare part group.

Table 4: Average accuracy values per hybrid classification algorithms.

\begin{tabular}{|c|c|c|c|c|c|c|c|}
\hline \multicolumn{3}{|c|}{ Apriori (min_confidence=0.7) } & \multicolumn{3}{c|}{ Apriori (min_confidence=0.7) } \\
and IkNN \\
\hline and AkN
\end{tabular}

According to the runtime analysis given in Figure 10, while Apriori causality rules within $[0.78,0.82]$ precision interval are intensively used, IkNNwC variation has a better prediction performance. Although increments at dynamic $k(\mathrm{DK})$ argument has a positive effect at the recall values of $\mathrm{IkNNwC}$ variation, the inverse effect is valid for AkNN and IkNNwAS variations. This is due to the fact that, while count-based adaptation at $\mathrm{kNN}$ is seemingly more robust to the changes made at the extents of neighborhood region, the selective prediction capabilities of average score-based adaptations are more vulnerable to these changes. Hence, the discriminative power of minor class is lost.
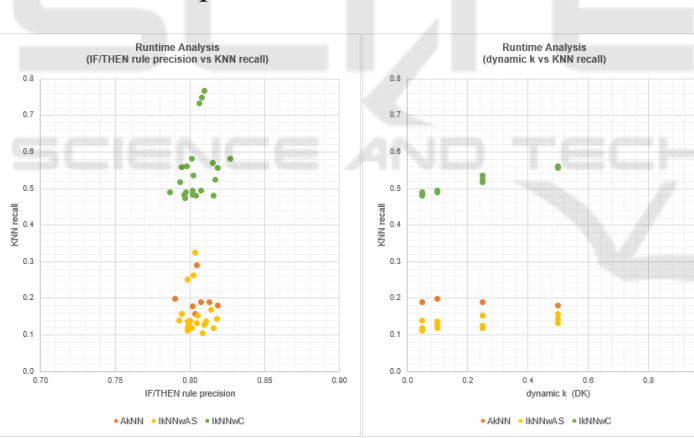

Figure 10: Runtime analysis for kNN adaptations.

Table 5: Average accuracy per spare part group. Average accuracy of proposed approach is improved by the increments at the confidence threshold.

\begin{tabular}{l|ccc}
\multicolumn{4}{c}{ spare part groups } \\
\hline & ALL & TOP3 & TOP6 \\
\hline Apriori & $54.02 \%$ & $59.86 \%$ & $55.68 \%$ \\
\hline Apriori (min_conf.0.7) + IKNNWC & $77.93 \%$ & $85.20 \%$ & $79.24 \%$ \\
\hline Apriori (min_conf:0.8) + IKNNWC & $77.99 \%$ & $85.25 \%$ & $79.88 \%$ \\
\hline Apriori (min_conf:0.9) + IKNNWC & $78.98 \%$ & $86.13 \%$ & $80.47 \%$ \\
\hline Apriori (min_conf:1.0) + IKNNWC & $80.68 \%$ & $87.66 \%$ & $82.24 \%$
\end{tabular}

As the next iteration, the confidence threshold determined in Section 3.4.2 is incremented linearly within $[0.7,1.0]$ interval. As a result, the combination of Apriori rules (with min_confidence $=1.0$ ) with $\mathrm{IkNNwC}$ variation (with $k$-limit $=2$ and $n e i g h$ limit $=$ $0.2 \%$ arguments) reaches to an average accuracy of $80.68 \%$ as shown in Table 5 .

When recall values per spare part are separately analyzed, spare part 303113250 (with a frequency of $13.01 \%$ as shown in Figure 5) has a significant increase of $7.8 \%$ at its recall values as shown in Figure 11. Potentially, erroneous causality rules with consequent equal to 303113250 are intensively handed over by the predictions made by $\mathrm{IkNNwC}$ variation and this online learning schema is relatively more accurate. Similar mechanism is valid for spare part 303113320 with the highest frequency given in Figure 5. A $2.8 \%$ increase at the recall values of the corresponding spare part causes a significant increasing-return effect on accuracy as shown in Table 5.

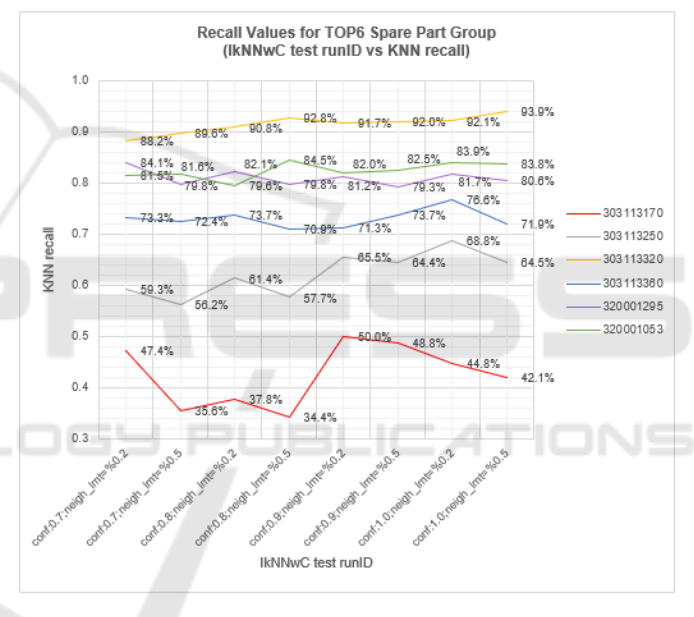

Figure 11: Recall values for TOP6 spare part group.

\subsection{Deployment}

Due to the results obtained at evaluation phase, the best runtime configuration is designated as Apriori causality rules (with $\min$ confidence $=1.0$ ) with IkNNwC variation (with $k$-limit $=2$ and neigh limit $=$ $0.2 \%$ arguments). This hybrid classification model is implemented as a custom function at SAP BW system as shown in Figure 12. In addition to spare part prediction, the underlying function recommends potential concomitant spare part consumptions. These associated consumptions are based on the association rules generated by alternative data exploration procedure stated in Section 3.2.2.

According to performance measurement, average prediction duration of a single fault incident is approximately 7.79 second (i.e. remote function 
connection (RFC) time between SAP CRM and BW systems is excluded). Since mean arrival time between two consecutive fault incidents is approximately 36.5 second, it is technically feasible to perform a real-time spare part prediction.

\begin{tabular}{|c|c|c|}
\hline \multicolumn{2}{|c|}{ Structure Editor: Display ET_RESULT from Entry } & \multirow[t]{2}{*}{1} \\
\hline 思4 & 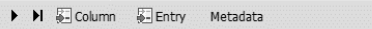 & \\
\hline \multicolumn{3}{|c|}{4 4 Entries } \\
\hline HZMBSLK & PRED_RESULI & 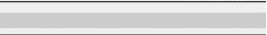 \\
\hline \begin{tabular}{|l|l|}
8049148769 \\
80094187769 \\
8049148769 \\
8049148769
\end{tabular} & 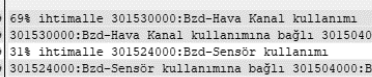 & $\begin{array}{l}\text { Bzd-Elekt.Kart tüketimi ( } 828 \text { güven) } \\
\text { Elekt.Kart tüketimi (32s güven) }\end{array}$ \\
\hline
\end{tabular}

Figure 12: View of spare part prediction result list. While odd numbered lines at PRED_RESULT prediction result list inform spare part predictions, even lines indicate concomitant spare part consumptions. HZMBSLK is the unique identifier for the corresponding fault incident.

\section{CONCLUSIONS}

This paper proposes a hybrid classification algorithm for the underlying spare part prediction scenario such that, while Apriori is adapted to handle data anomalies and redundancies emerged at data exploration, significance weights obtained at C4.5 incorporates the inter-dimension similarity at interpreting the neighborhood among fault instances. Finally, two adaptations of weighted $\mathrm{kNN}$ are applied: IkNNwC gives more importance to major instances that are more likely to represent a dominant class in neighborhood region of feature space, IkNNwAS aims to balance the discriminative power of minor class.

According to experimental results, proposed hybrid classification algorithm doubles the humanlevel performance at spare part prediction, which is approximately $40 \%$ accuracy. This performance implies a $50 \%$ decrease at the average number of customer visits per fault incident. Hence a significant cutting at especially sales and distribution costs is expected by the effect of spare part prediction model. As future work, we plan to extend the corresponding modeling to other product groups.

\section{REFERENCES}

Aguilera, J., Gonzalez, L. C., Montes-y-Gomez, M. \& Rosso, P. (2019). A New Weighted k-Nearest Neighbor Algorithm based on Newton's Gravitational Force. CIARP 2018, 305-313.

Boriah, S., Chandola, V., \& Kumar, V. (2008). Similarity Measures for Categorical Data: A Comparative Evaluation, SDM, 243-254.
Chapman, P., Clinton, J., Kerber, R., Khabaza, T., Reinartz, T., Shearer, C., Wirth, R. (1999). CRISP-DM 1.0 Stepby-Step Data Mining Guide.

Domingos, P. (2015). The Master Algorithm: How the Quest for the Ultimate Learning Machine Will Remake Our World. Basic Books.

Dudani, S.A. (1976). The Distance-Weighted k-NearestNeighbor Rule. IEEE Trans. Syst. Man Cybern. SMC6(4), 325-327.

Kantardzic, M. 2011). Data Mining: Concepts, Models, Methods, and Algorithms. Wiley-IEEE Press.

Liu, C., Cao, L. \& Yu, P. S. (2014). A Hybrid Coupled kNearest Neighbor Algorithm on Imbalance Data. International Joint Conference on Neural Networks (IJCNN).

Liu, W. \& Chawla, S. (2011). Class Confidence Weighted kNN Algorithms for Imbalanced Data Sets, Advances in Knowledge Discovery and Data Mining, 345-356.

Mateos-Garcia, D., Garcia-Gutierrez, J. \& RiquelmeSantos, J.C. (2016). An Evolutionary Voting For kNearest Neighbors. Expert Syst. Appl. 43, 9-14.

Pang-Ning, T., Steinbach, M. \& Kumar, V. (2006). Introduction to Data Mining, Library of Congress, 74.

Parvinnia, E., Sabeti, M., Zolghadri Jahromi, M. \& Boostani, R. (2014). Classification of EEG Signals Using Adaptive Weighted Distance Nearest Neighbor Algorithm. J. King Saud Univ. Comput. Inf. Sci. 26(1), 1-6.

Song, Y., Huang, J., Zhou, D., Zha, H. \& Giles, C. L. (2007). IkNN: Informative K-Nearest Neighbor Pattern Classification, Knowledge Discovery in Databases: PKDD 2007, 248-264.

Tan, S. (2005). Neighbor-Weighted K-Nearest Neighbor for Unbalanced Text Corpus. Expert Syst. Appl. 28(4), 667-671.

Wang, C., Cao, L., Wang, M. Li, J., Wei, W. \& Ou, Y. (2011). Coupled Nominal Similarity in Unsupervised Learning, CIKM 2011, 973-978.

Yuxuan, L. \& Zhang, X. (2011). Improving $K$ Nearest Neighbor with Exemplar Generalization for Imbalanced Classification, 15th Pacific-Asia Conference, PAKDD 2011, 1-12.

Zhang, S., Cheng, D., Deng, Z., Zong, M. \& Deng, X. (2017). A Novel kNN Algorithm with Data-Driven $k$ Parameter Computation. Pattern Recognit. Lett. 1-11.

Zhu, Q., Feng, J. \& Huang, J. (2016). Natural Neighbor: A Self-Adaptive Neighborhood Method without Parameter k. Pattern Recognit. Lett. 80, 30-36. 